\title{
Promoting Physical Activity and Executive Functions Among Children: A Cluster Randomized Controlled Trial of an After-School Program in Australia
}

\author{
Sanne L.C. Veldman, Rachel A. Jones, Rebecca M. Stanley, Dylan P. Cliff, Stewart A. Vella, \\ Steven J. Howard, Anne-Maree Parrish, and Anthony D. Okely
}

\begin{abstract}
Background: The aim of this study was to examine the efficacy of an embedded after-school intervention, on promoting physical activity and academic achievement in primary-school-aged children. Methods: This 6-month, 2-arm cluster randomized controlled trial involved 4 after-school centers. Two centers were randomly assigned to the intervention, which involved training the center staff on and implementing structured physical activity (team sports and physical activity sessions for 75 min) and academic enrichment activities (45 min). The activities were implemented 3 afternoons per week for 2.5 hours. The control centers continued their usual after-school care practice. After-school physical activity (accelerometry) and executive functions (working memory, inhibition, and cognitive flexibility) were assessed pre- and postintervention. Results: A total of 60 children were assessed $(7.7[1.8] \mathrm{y} ; 50 \%$ girls) preintervention and postintervention (77\% retention rate). Children in the intervention centers spent significantly more time in moderate to vigorous physical activity (adjusted difference $=2.4 \%$; $95 \%$ confidence interval, 0.6 to $4.2 ; P=.026$ ) and scored higher on cognitive flexibility (adjusted difference $=1.9$ units; $95 \%$ confidence interval, 0.9 to $3.0 ; P=.009$ ). About $92 \%$ of the intervention sessions were implemented. The participation rates varied between $51 \%$ and 94\%. Conclusion: This after-school intervention was successful at increasing moderate to vigorous physical activity and enhancing cognitive flexibility in children. As the intervention was implemented by the center staff and local university students, further testing for effectiveness and scalability in a larger trial is required.
\end{abstract}

Keywords: academic enrichment, primary school, working memory, inhibition, and cognitive flexibility

Time spent in after-school programs (which can range from 3 to $6 \mathrm{PM}$ on school days) has been identified as a critical window for the promotion of physical activity and academic enrichment. ${ }^{1}$ This time period is typically characterized by high levels of physical inactivity and sedentary behavior ${ }^{2}$ and minimal academic enrichment opportunities, ${ }^{2-4}$ reducing its potential to impact these areas. An umbrella review, composed of 6 systematic reviews (20092016), examining the effects of after-school programs on child and adolescent physical activity and health found there was only modest support of the benefits of after-school programs on physical activity levels. ${ }^{5}$ Programs that were delivered within school settings twice or more per week showed greater potential in increasing children's physical activity; however, the breadth of factors influencing the effectiveness of after-school programs remained largely unknown. More research to understand what characterizes highquality after-school physical activity programs was recommended. Factors such as the use of theoretical frameworks and objective measures of physical activity and reporting the fidelity of interventions was suggested. 5

\footnotetext{
Veldman, Jones, Stanley, Cliff, Vella, Howard, Parrish, and Okely are with Early Start, University of Wollongong, Wollongong, NSW, Australia. Veldman is also with the Department of Public and Occupational Health, Amsterdam Public Health Research Institute, Amsterdam UMC, Vrije Universiteit Amsterdam, Amsterdam, The Netherlands. Jones, Stanley, Cliff, Parrish, Vella, Okely, and Howard are also with the Faculty of Social Sciences, University of Wollongong, Wollongong, NSW, Australia. Jones, Cliff, Vella, Parrish, and Okely are also with the Illawarra Health and Medical Research Institute, University of Wollongong, Wollongong, NSW, Australia. Okely (tokely@uow.edu.au) is corresponding author.
}

In addition to after-school programs focusing on increasing physical activity among children, studies have also reported other outcomes, such as academic enrichment. A meta-analysis examined the effects of after-school programs on personal and social skills, including academic performance. ${ }^{3}$ Academic performance was assessed mostly through achievement test scores ( $n=20$ studies), grades ( $n=25$ studies), and school attendance $(n=21$ studies). The results showed that both test scores and grades increased for students attending after-school programs that included the presence of 4 recommended practices (SAFE: sequenced, active, focused, and explicit) compared with students who did not attend these programs. ${ }^{3}$ In addition, studies have recommended the use of evidence-based instructional strategies, a supportive environment for learning activities, conducting preassessments to ensure programs are meeting academic needs, and coordinating tutoring with school curricula. ${ }^{3,6}$

With increasing numbers of children attending after-school programs in Australia, ${ }^{7}$ there is a need for programs to promote physical activity and provide academic enrichment. In response, the authors designed an afterschool program that combined physical activity and academic enrichment activities. This program was piloted in a randomized controlled trial, ${ }^{8}$ and positive results for physical activity were reported. However, academic/learningrelated outcomes were not assessed. Furthermore, the program was facilitated by researchers, limiting program sustainability and transferability. An important part of translational research includes the continued use of programs to sustain achievement of desirable goals and outcomes. ${ }^{9}$

To address the limitations in the current literature and achieve sustainability of programs, it is also important to examine the 
feasibility and acceptability of programs' implementation by the setting staff. Therefore, the primary aim of this study was to examine the efficacy of an embedded after-school intervention consisting of training and supporting staff and structured physical activity and academic enrichment activities on promoting physical activity in primary-school-aged children. The secondary aims included examining the efficacy of the intervention on academic achievement (executive functions) and examining the feasibility and acceptability of the intervention.

\section{Methods}

\section{Design, Participants, and Setting}

This 6-month, 2-arm cluster randomized controlled trial involved 4 after-school care centers located in New South Wales, Australia. The centers were pair-matched based on physical space, the number of children, and the socioeconomic status of the enrolled children's parents. All children aged between 5 and 10 years who attended at least twice per week were invited to participate following parental consent. Children were excluded from the study if they were diagnosed with a medical condition limiting their engagement in physical activities (eg, cerebral palsy). Following the baseline measures, each pair of centers was randomized into either the intervention group $(n=2)$ or control group $(n=2)$ by drawing the allocation out of a hat.

The intervention centers participated in the intervention between April and September 2014, while the control centers continued their usual after-school care practice. The study was approved by the Human Ethics Research Committee at the University of Wollongong (HE14/067). The parents/caregivers provided written consent, and the children provided assent before the data collection. The reporting was done following the CONSORT statement. $^{10}$

\section{Intervention}

The intervention involved both training and supporting setting staff and university students, as well as a physical activity and academic enrichment intervention for 5- to 10-year-old children. The intervention was guided by social cognitive theory and underpinned by the 4 key processes for learning and adapting new behaviors: attention, retention, production, and motivation. ${ }^{11}$ The activities were designed to (1) include stimulus material and specific lesson activities to engage and direct the attention of the educators and children, (2) match children's cognitive and behavioral skill levels and provide opportunities to enhance mastery experiences, and (3) emphasize perceived choice and control, as well as personalization, contextualization, challenge, curiosity, and mastery through activities that enhance intrinsic motivation, greater persistence, better performance, and higher satisfaction.

The staff from the intervention centers and undergraduate students (Bachelor of Primary Education, referred to hereafter as student instructors) from the University of Wollongong received 4 hours of training prior to the commencement of the intervention. This training was delivered over several sessions at the University of Wollongong. The training covered the following components: how to facilitate structured physical activity sessions using good principles and practices to maximize participation and motivation, skill development, minimizing wait times, and availability of equipment by applying the $7 \mathrm{C}$ 's of intrinsic motivation. ${ }^{12}$ Specific content was provided for the team sports and physical activity sessions covered in the intervention. Demonstrations of activities were provided by the research team, followed by a discussion of the aforementioned topics. Student instructors were recruited through a compulsory fourth-year undergraduate subject (EDSE401) in which they were required to complete a minimum of 30 hours of service in a local community-based organization. The students were not graded on their participation or contribution. Once they completed their compulsory 30 hours of service, they were paid by the research project for any remaining hours they were involved in the intervention.

The intervention was implemented 3 afternoons per week for 2.5 hours by the center staff and student instructors $(n=13)$. The student instructors rotated through the intervention, with 2 present at each session. The intervention had 2 components: team sports and physical activity sessions (75 min) and academic enrichment activities $(45 \mathrm{~min}$ ). The sessions were composed of 1 type of team sport being played per session, rotating between soccer, netball, basketball, and touch/oztag or a thematic session based on popular TV shows, such as The Amazing Race and Survivor. These sessions included backyard games, bootcamp, and physical challenges. All activities were designed to be fun and challenging (both tactically and physiologically), aiming to maximize students' motivation to participate. The academic enrichment activities involved children completing their prescribed homework, with staff and university students acting as tutors where needed (including 20 min reading).

\section{Measurements}

To determine the efficacy of the intervention, children were assessed preintervention and postintervention (6 mo after baseline) by trained data collectors blinded to the group allocation on the outcomes of physical activity and executive functions. Physical activity was measured using accelerometers (Actigraph, Pensacola, FL). Children wore the device on their right hip for a week during the after-school program. The monitor was put on when children arrived at the after-school program and removed when they left. "On-off" times were reported by the data collectors in a log. An epoch of $30 \mathrm{~Hz}$ was used, and files were reintegrated into 15-second epochs. Nonwear time was defined as 20 minutes of consecutive zeros, and children had to wear the accelerometer on at least one after-school day. Physical activity was analyzed and classified using the Evenson cut points recommended for primary-schoolaged children: time spent in sedentary time $(\leq 100$ counts per minute), light-intensity physical activity (101-2295 counts per minute), moderate-intensity physical activity (2296-4011 counts per minute), and vigorous-intensity physical activity ( $\geq 4012$ counts per minute). ${ }^{13}$ To account for variability in the number of days monitored and daily wear times, physical activity outcomes were averaged over the monitoring days and the percentage of time spent in different behaviors were used for analysis. Analysis of the accelerometer data were conducted using the (ActiLife, Pensacola, FL) software.

Executive functions were assessed using 3 executive function measures from the iPad-based Early Years Toolbox, ${ }^{14}$ assessing working memory (Mr. Ant), inhibition (Go/No-Go), and shifting (Card-Sorting). These measures were designed to be brief ( $\sim 5 \mathrm{~min})$ and engaging. In Mr Ant, working memory was assessed by asking the children to remember the spatial locations of an increasing number of stickers placed on a cartoon ant, and then to identify these locations after a brief retention interval. For Go/No-Go, inhibition was assessed by tapping the screen on go trials ("catch the fish") and withholding this response on no-go trials ("avoid the 
sharks"). Shifting was assessed by children sorting stimuli by one sorting rule (eg, shape), switching to the alternate rule (eg, color) and, if a performance threshold was met, then flexibly switching between these rules. The early years toolbox has good reliability (Cronbach $\alpha>.80$ ) in children up to 6 years old. As raw scores were used for the data analysis, the assessment tool was also deemed appropriate for the study sample. The iPad measures were conducted in a quiet space facilitated by a trained data collector.

Process data were collected to assess feasibility and acceptability. Feasibility was assessed using recruitment and retention rates, and acceptability was based on participation rates, the number of sessions implemented, and an informal interview with the student instructors upon completion of the intervention. Participation was recorded, along with daily attendance and the reasons for nonparticipation. The participation rate was calculated based on the activity participation by the attending enrolled children. Delivery rates were reported by the center staff and student instructors. The student instructors participated in an informal interview to assess the acceptability of the intervention. They were asked questions about their experience of being involved in the intervention, positive and negatives aspects of the intervention implementation, and their perceived impact of the intervention on the children. The aim was to recruit $90 \%$ of eligible children, retain $80 \%$ of the participants at follow-up, and deliver $90 \%$ of the intervention sessions, with a participant rate of $75 \%$.

\section{Data Analysis}

Statistical analyses were conducted using (STATA, College Station, TX) (version 15). ${ }^{15}$ Descriptive statistics (mean [SD]) were used to describe participant characteristics, physical activity, and executive function outcomes, preintervention and postintervention and per group (intervention and control). The intervention effects were assessed by comparing the follow-up outcomes between the control and intervention group, using generalized linear models. All models were adjusted for clustering using survey logistic procedures and included sex, age, and baseline values as covariates. The significance level was set at $P<.05$.

\section{Results}

A total of 60 children (mean age $=7.7$ [1.8] y; 50.0\% girls, $n=30$ intervention and $\mathrm{n}=30$ control) completed all measurements at preintervention and postintervention. At the baseline, the children spent $63.2 \%$ of their time in after-school care in sedentary time, $21.1 \%$ in light-intensity physical activity, and $15.7 \%$ in moderate to vigorous physical activity (MVPA; see Table 1). On average, they scored 3.58 (1.71) on the working memory assessment (range 0-8), $0.84(0.17)$ on the inhibition assessment (range $0-1$ ), and 15.28 (5.70) on the shifting assessment (range 0-18). All baseline values were similar for the intervention and control centers. At follow-up, the children in the intervention centers spent significantly more time in MVPA (adjusted mean difference $=2.4 \%$; $95 \%$ confidence interval, 0.6 to $4.2 ; P=.026$ ) compared with those in the control group. The children in the intervention group scored significantly higher on the shifting assessment (adjusted mean difference $=1.9 ; 95 \%$ confidence interval, 0.9 to $3.0 ; P=.009$ ) of the executive functions test compared with children in the control group (Table 2).

Feasibility and acceptability of the intervention were examined using process data. Seventy-eight children were recruited to the intervention, and $76.9 \%$ were retained $(n=60)$. The recruitment rates could not be determined due to missing data. Reasons for nonretention were children being absent during the follow-up measurements $(n=7)$, leaving the center $(n=6)$, not wanting to participate in the intervention anymore $(n=3)$, changing attendance, and thereby no longer meeting the requirement of attendance at least 2 days per week $(n=1)$, or an unrelated medical reason (broken arm; $n=1$ ) (see Figure 1 ).

Overall, 92\% (88/96) of the intervention sessions were implemented. Adverse weather $(n=1)$, other university commitments for the student instructors (eg, examinations) $(n=4)$, or public holidays $(n=3)$ resulted in the intervention not being facilitated. Of the implemented team sports and physical activity sessions, 55 sessions were implemented as planned (eg, at least $75 \mathrm{~min}$ ), 19 sessions did not meet the planned 75 minutes, and 14 sessions had missing data regarding total duration. The student instructors suggested that the intervention was not implemented completely as planned on fewer than 3 occasions, as the children were less motivated to participate, as they "had a rough day at school or home and did not want anything really structured". The participation rates varied between the 2 intervention centers: $75.2 \%$ to $94.25 \%$ for intervention center 1 and $51.1 \%$ to $65.9 \%$ for intervention center 2 . Children not formally enrolled in the study but attending the afterschool program also participated in the intervention. The number of additional children ranged from 2 to 14 per day, but these are not included in the participation rates. Participant engagement was

\section{Table 1 Descriptives at Baseline}

\begin{tabular}{|c|c|c|c|c|}
\hline & Total $(\mathrm{N}=60)$ & Intervention $(n=30)$ & Control $(n=30)$ & $\mathbf{P}^{\mathbf{a}}$ \\
\hline \multicolumn{5}{|l|}{ Demographics } \\
\hline Age (mean [SD]) & $7.73(1.84)$ & $7.62(1.83)$ & $7.84(1.87)$ & .650 \\
\hline Sex (n, $\%$ of girls) & $30,50.00$ & $14,46.70$ & $16,53.30$ & $.606^{\mathrm{b}}$ \\
\hline \multicolumn{5}{|l|}{ Physical activity } \\
\hline$\% \mathrm{ST}(\% \pm \mathrm{SD})$ & $63.20(11.19)$ & $62.06(11.99)$ & $64.34(10.40)$ & .435 \\
\hline$\%$ Light $(\% \pm \mathrm{SD})$ & $21.06(5.44)$ & $21.91(6.20)$ & $20.20(4.50)$ & .226 \\
\hline$\% \operatorname{MVPA}(\% \pm \mathrm{SD})$ & $15.74(7.16)$ & $16.02(7.33)$ & $15.45(7.10)$ & .796 \\
\hline \multicolumn{5}{|l|}{ Executive functions } \\
\hline Working memory (range $0-8$ ) & $3.58(1.71)$ & $3.36(1.56)$ & $3.80(1.86)$ & .318 \\
\hline Inhibition (range $0-1$ ) & $0.84(0.17)$ & $0.88(0.12)$ & $0.80(0.20)$ & .052 \\
\hline Shifting (range $0-18$ ) & $15.28(5.70)$ & $15.27(6.07)$ & $15.30(5.41)$ & .982 \\
\hline
\end{tabular}

Abbreviations: MVPA, moderate to vigorous physical activity; ST, sedentary time.

${ }^{\mathrm{a}}$ Independent samples $t$ test, ${ }^{\mathrm{b}} \mathrm{Chi}$-squared test. 
Table 2 Summary of Results for Primary and Secondary Outcomes

\begin{tabular}{|c|c|c|c|c|c|c|c|c|}
\hline & \multicolumn{2}{|c|}{ Baseline } & \multicolumn{2}{|c|}{ Follow-up } & \multirow{2}{*}{$\begin{array}{c}\text { Adjusted } \\
\text { mean } \\
\text { difference }\end{array}$} & \multirow[b]{2}{*}{$95 \% \mathrm{Cl}$} & \multirow[b]{2}{*}{$P$} & \multirow[b]{2}{*}{ Cohen $d$} \\
\hline & $\begin{array}{l}\text { Intervention } \\
(n=30)\end{array}$ & $\begin{array}{l}\text { Control } \\
(n=30)\end{array}$ & $\begin{array}{l}\text { Intervention } \\
(n=30)\end{array}$ & $\begin{array}{l}\text { Control } \\
(n=30)\end{array}$ & & & & \\
\hline \multicolumn{9}{|l|}{ Physical activity } \\
\hline$\% \mathrm{ST}(\% \pm \mathrm{SD})$ & $62.06(11.99)$ & $64.34(10.40)$ & $61.86(13.00)$ & $67.51(11.30)$ & -3.91 & -9.60 to 1.79 & .117 & 0.45 \\
\hline$\%$ Light $(\% \pm \mathrm{SD})$ & $21.91(6.20)$ & $20.20(4.50)$ & $21.63(6.42)$ & 19.04 (4.67) & 1.58 & -1.93 to 5.09 & .247 & 0.46 \\
\hline$\% \operatorname{MVPA}(\% \pm \mathrm{SD})$ & $16.02(7.33)$ & $15.45(7.10)$ & $16.51(7.87)$ & $13.44(7.43)$ & 2.39 & 0.55 to 4.22 & .026 & 0.40 \\
\hline \multicolumn{9}{|l|}{ Executive functions } \\
\hline $\begin{array}{l}\text { Working memory } \\
\text { (range } 0-8 \text { ) }\end{array}$ & $3.36(1.56)$ & $3.80(1.86)$ & $3.70(2.20)$ & $3.47(1.82)$ & 0.59 & -0.91 to 2.08 & .299 & 0.11 \\
\hline $\begin{array}{l}\text { Inhibition } \\
\text { (range 0-1) }\end{array}$ & $0.88(0.12)$ & $0.80(0.20)$ & $0.85(0.13)$ & $0.75(0.20)$ & 0.05 & -0.04 to 0.14 & .183 & 0.59 \\
\hline $\begin{array}{l}\text { Shifting } \\
\text { (range 0-18) }\end{array}$ & $15.27(6.07)$ & $15.30(5.41)$ & $17.67(5.92)$ & $15.87(6.16)$ & 1.93 & 0.91 to 2.96 & .009 & 0.30 \\
\hline
\end{tabular}

Abbreviations: CI, confidence interval; MVPA, moderate to vigorous physical activity; ST, sedentary time.

enhanced when the student instructors were involved in the facilitation of the sessions compared with only the setting staff. One student instructor commented, "The children really enjoyed the input and engagement of us as staff members, I found that when we got involved, the activities ran a lot more smoothly and effectively ... ." Similarly, another student instructor commented, "Motivation was mostly enhanced when us as instructors got involved and motivated the students to have a go ... ."

Overall, the student instructors suggested that the intervention was successful. They suggested that the center staff were "extremely supportive" and that working with them as a team of instructors was a positive experience. One student instructor summarized the impact of the intervention on the children:

"There was a great deal of involvement from [the children]. I believe overall the intervention did make a difference to the children's lives, they were so excited each week to participate and some of the feedback from the parents was how the children had been teaching their little cousins or friends these activities at home as well. The tutoring aspect was a highly effective section of the intervention. It was a great opportunity to form closer relationships with children. Those children who regularly took part in the intervention had lots of fun and formed great relationships with the university student coaches."

\section{Discussion}

The authors found that training and supporting the staff to implement an after-school physical activity and academic enrichment intervention is an effective, feasible, and acceptable way to increase time spent in MVPA and improve some executive functions.

This study reported significant differences in the time spent in MVPA between the intervention group and the control group. A previous systematic review has specifically examined the effects of after-school programs on MVPA. ${ }^{16}$ Of the 25 included studies, 12 studies used accelerometers to assess physical activity, and only 6 studies were eligible for meta-analysis. The results of the review and meta-analysis showed there is mixed evidence supporting the effectiveness of after-school programs and highlighted the large variability in study designs, making comparisons difficult. The pooled intervention effect at the end-point follow-up was 4.84 minutes per day of MVPA (95\% confidence interval, -0.94 to 10.61 ).
There are several explanations for the positive results found in this study. Recent evidence-based recommendations for increasing physical activity include, but are not limited to, allocating at least 60 minutes per day to physical activity opportunities ${ }^{17}$; organized physical activity opportunities ${ }^{17,18}$; and positive teacher behaviors, attitudes, and interactions. ${ }^{18,19}$ The current intervention included 75 minutes of organized physical activity opportunities. The physical activities offered were structured and were designed to be engaging, inclusive, and motivating, and utilized different equipment. They aligned with the best evidence-based practices in this area, such as the LET US Play principles, ${ }^{20}$ which include removing lines; limiting elimination; small team sizes; motivating uninvolved staff and children; and modifying the space, equipment, and rules, with the specific aim to engage children in MVPA for at least $50 \%$ of the organized activity. This may help explain the changes in MVPA. In addition, the motivation of the center staff and the student instructors may have contributed to the higher levels of MVPA in the intervention group compared with the controls. The center staff and the student instructors were trained in applying motivation principles in their implementation of activities.

Significant differences were reported for shifting. The positive result found for shifting can be explained by a potential stronger challenge to shifting in the physical activity and other executive function scores (eg, inhibition) approaching the ceiling at the baseline, thereby leaving less opportunity to detect positive changes. During physical activity and sports, you need to shift between opponents, strategies (eg, offense to defense), and planning (eg, current situations and thinking ahead).

Even though the exact recruitment rates could not be determined because of missing data, recruitment was still a component of feasibility and, as such, will be discussed. Several children who participated in the intervention were not a part of the study, meaning that recruitment can be improved. Recruitment could have been influenced by a number of factors. Children were provided with information sheets and consent forms and were asked to give them to their parents. Although a number of reminders were given to the children, the exact number of information sheets and consent forms given to parents is unknown. Furthermore, given the nature of after-school programs, parental contact is generally minimal; thus, it was difficult to discuss the intervention with the parents face-to-face and inform them of the 


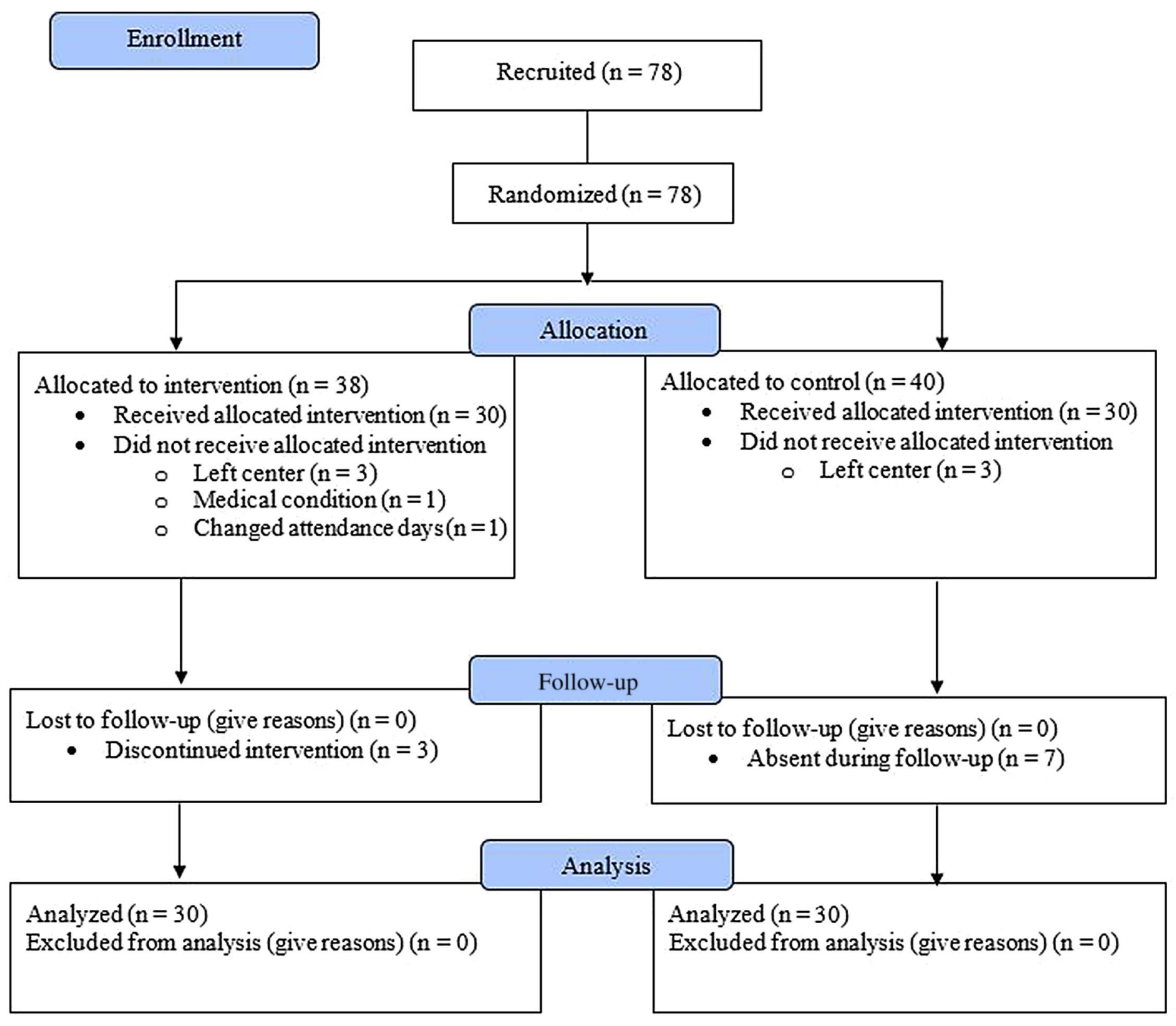

Figure 1 - CONSORT 2010 flow diagram.

potential benefits of the intervention or answer any questions that they might have had about the intervention. Recruitment rates may have been enhanced by advertising the intervention in school newsletters, having the school principal endorse the intervention, or further highlighting the academic enrichment activities in the intervention, as parents may be interested in the benefits of such activities. A trial session may have also been helpful, as this would have provided an opportunity for children to experience some of the activities and meet and greet the student instructors. The retention rate was slightly lower than expected, although some reasons were beyond the control of the center staff (eg, change of after-school days due to family circumstances or children leaving the center).

Overall, the intervention was found to be highly acceptable (high intervention fidelity and positive comments from student instructors); however, the participation rates do warrant further investigation. The participation rates varied between intervention centers, as well as between days of the intervention and throughout the intervention. The significant variation in the participation rates between intervention centers is difficult to explain, given that identical interventions were facilitated at both intervention sites and the center staff and student instructors received identical training and equipment. Anecdotally, session participation tended to peak during the first 30 minutes of the session and then declined. The session participation rates could also have been influenced by the types of activities offered on the day and the ratio of boys and girls participating in the activities. However, no attendance data were collected during different stages of the sessions, nor the ratio of boys and girls participating to examine this further. Children's enrollment was family specific, and the number and mix of children 
changed daily. Therefore, the mix of children at the different sites may have influenced the participation rate. Typically, children in Australia do not attend after-school programs with their central peer group and, in the absence of their regular peer group, may have found participating in group-based games more daunting. The physical activities included in the intervention were designed to be engaging, highly motivating, and appropriate for boys and girls of all abilities. However, a critical mass is typically needed for the success of some team-based physical activities; thus, it would be important to ensure that the teams are of appropriate numbers. A review of the activities provided may also be necessary, although these activities have been trialed previously and have been found to be highly appropriate. ${ }^{8}$

The length of the session ( $75 \mathrm{~min}$ ) may need to be reviewed to ensure maximum participation in the entire session. A shortened session of about 30 minutes or 2 small sessions may be more appropriate. These options would mean that the participation rate would not be affected by children being picked up early by their parents. Furthermore, one intervention center had a larger outdoor space and better outdoor lighting, which was more conducive to playing outside for longer periods of time. Although the participation rates varied, a significant proportion of children not formally enrolled in the study participated in the intervention. This was encouraging and suggested that the intervention was inclusive and "looked" interesting and fun and was something that other children wanted to participate in.

This was the first known intervention to assess the effects of an after-school intervention on physical activity and executive functions. The intervention was successfully implemented several times a week over several months. Longer studies are recommended to maximize transferability, sustainability, and maintenance of outcomes. This includes building on the current project by scaling up this model through an effectiveness or translational trial.

The intervention successfully resulted in strong partnerships between the center staff and university student instructors. Ongoing collaborations between partner organizations and universities could be one important avenue for high-impact interventions. Future avenues to strengthen these partnerships and ensure they are sustainable should be investigated. A promising aspect of the intervention was that all university students were recruited from a subject that required students to complete a number of hours of community service. Such requirements are common in most universities. As such, this approach has the potential for scalability in areas where universities are located and after-school programs exist. However, the results need to be considered in light of the following limitations. The study had a small sample size, which may have impacted the ability to detect significant effects for secondary outcomes. The participation rates varied between centers and days and within sessions. Due to the latter, monitoring of exact participation rates was difficult, and more detailed information would have been helpful. The assessment tool used for executive functions has not been validated in the age group of the current study, yet, as norms were not used, the tool was deemed appropriate. Physical activity was only measured during the afterschool period, whereas it would be interesting to examine the effects of an afterschool intervention on whole-day activity levels. The study was conducted in collaboration with one after-school-care organization; additional studies should be conducted to ensure that this model is transferrable for other organizations. Measuring cognitive outcomes and developing a partnership with organizations is recommended to examine the impact of the after-school programs on academic achievement.

\section{Conclusion}

This study successfully embedded a structured physical activity and academic enrichment intervention in an after-school care setting. The intervention was successful at increasing physical activity, and it provides the novel evidence that such an intervention can enhance children's executive functions. With a high and increasing number of children attending after-school programs, this is an important setting to consider when targeting both health and developmental outcomes. This intervention and implementation model provides an evidence-based approach that has the potential to be further tested in community settings. It proposes a potentially sustainable partnership model for universities and their local communities.

\section{Acknowledgments}

The authors would like to thank Big Fat Smile for their support of this project, in particular, Chris Tulloch and Cinzia Immuni. The authors would like to thank Tamara Raso for helping with project management and the undergraduate students from the University of Wollongong who were involved as the volunteer coaches. This project was funded through the University of Wollongong Research Partnerships Grant Scheme. The authors have no competing interests to declare in relation to this project. Australian New Zealand Clinical Trials Registry (ANZCTR)—ACTRN: ACTRN12614000925640

\section{References}

1. Atkin AJ, Gorely T, Biddle SJ, Marshall SJ, Cameron N. Critical hours: physical activity and sedentary behavior of adolescents after school. Pediatr Exerc Sci. 2008;20(4):446-456. PubMed ID: 19168921 doi:10.1123/pes.20.4.446

2. Trost SG, Rosenkranz RR, Dzewaltowski D. Physical activity levels among children attending after-school programs. Med Sci Sports Exerc. 2008;40(4):622-629. PubMed ID: 18317385 doi:10.1249/ MSS.0b013e318161eaa5

3. Durlak JA, Weissberg RP, Pachan M. A meta-analysis of after-school programs that seek to promote personal and social skills in children and adolescents. Am J Community Psychol. 2010;45(3-4):294-309. PubMed ID: 20300825 doi:10.1007/s10464-010-9300-6

4. Giles CM, Kenney EL, Gortmaker SL, et al. Increasing water availability during afterschool snack: evidence, strategies, and partnerships from a group randomized trial. Am J Prev Med. 2012;43(suppl 3):S136S142. PubMed ID: 22898163 doi:10.1016/j.amepre.2012.05.013

5. Demetriou Y, Gillison F, McKenzie TL. After-school physical activity interventions on child and adolescent physical activity and health: a review of reviews. Adv Phys Educ. 2017;7(2):191-215. doi:10.4236/ape.2017.72017

6. Birmingham J, Pechman EM, Russell CA, Mielke M. Shared features of high-performing after-school programs: A follow-up to the TASC evaluation. Washington, DC: Policy Studies Associates; 2005.

7. ABS. 4402.0 Childhood Education and Care. Canberra: Australian Bureau of Statistics; 2017.

8. Jones RA, Kelly J, Cliff DP, Batterham M, Okely AD. Acceptability and potential efficacy of single-sex after-school activity programs for overweight and at-risk children: the Wollongong SPORT RCT. Pediatr Exerc Sci. 2015;27(4):535-545. PubMed ID: 26305240 doi:10.1123/pes.2015-0116

9. Shelton RC, Cooper BR, Stirman SW. The sustainability of evidencebased interventions and practices in public health and health care. 
Annu Rev Public Health. 2018;39(1):55-76. PubMed ID: 29328872 doi:10.1146/annurev-publhealth-040617-014731

10. Schulz KF, Altman DG, Moher D. CONSORT 2010 statement: updated guidelines for reporting parallel group randomised trials. Int J Surg. 2011;9(8):672-677. PubMed ID: 22019563 doi:10.1016/j. ijsu.2011.09.004

11. Bandura A. Social foundations of thought and action: A social cognitive theory. Englewood Cliffs, NJ: Prentice-Hall; 1986.

12. Lepper M, Master A, Yow W. Intrinsic motivation in education. Adv Motiv Achiev. 2008; 15:521-555.

13. Evenson KR, Catellier DJ, Gill K, Ondrak KS, McMurray RG. Calibration of two objective measures of physical activity for children. J Sports Sci. 2008;26(14):1557-1565. PubMed ID: 18949660 doi:10.1080/02640410802334196

14. Howard SJ, Melhuish E. An early years toolbox for assessing early executive function, language, self-regulation, and social development: validity, reliability, and preliminary norms. J Psychoeduc Assess. 2017;35(3):255-275. PubMed ID: 28503022 doi:10.1177/ 0734282916633009

15. Stata Statistical Software: Release 13 [computer program]. TX: StataCorp LP; 2015.
16. Mears R, Jago R. Effectiveness of after-school interventions at increasing moderate-to-vigorous physical activity levels in 5- to 18-year olds: a systematic review and meta-analysis. $\mathrm{Br} J$ Sports Med. 2016;50(21):1315-1324. PubMed ID: 27222308 doi:10.1136/ bjsports-2015-094976

17. Brazendale K, Beets MW, Weaver RG, Huberty J, Beighle AE, Pate RR. Wasting our time? Allocated versus accumulated physical activity in afterschool programs. J Phys Act Health. 2015;12(8): 1061-1065. PubMed ID: 25271393 doi:10.1123/jpah.2014-0163

18. Weaver RG, Beets MW, Huberty J, Freedman D, Turner-Mcgrievy G, Ward D. Physical activity opportunities in afterschool programs. Health Promot Pract. 2015;16(3):371-382. PubMed ID: 25586132 doi: $10.1177 / 1524839914567740$

19. Behrens TK, Miller DJ, Schuna JM Jr, Liebert ML. Physical activity intensity, lesson context, and teacher interactions during an unstructured afterschool physical activity program. J Sch Health. 2015;85(12):880-885. PubMed ID: 26522177 doi:10.1111/josh. 12345

20. Brazendale K, Chandler JL, Beets MW, et al. Maximizing children's physical activity using the LET US Play principles. Preventive Medicine. 2015;76:14-19. PubMed ID: 25862947 doi:10.1016/j. ypmed.2015.03.012 